\title{
ERO1 $\alpha$-dependent endoplasmic reticulum-mitochondrial calcium flux contributes to ER stress and mitochondrial permeabilization by procaspase-activating compound-1 (PAC-1)
}

\author{
M Seervi ${ }^{1}$, PK Sobhan ${ }^{1}$, J Joseph ${ }^{1}$, K Ann Mathew ${ }^{1}$ and TR Santhoshkumar ${ }^{* 1}$
}

Procaspase-activating compound-1 (PAC-1) is the first direct caspase-activating compound discovered; using an in vitro cellfree system of caspase activation. Subsequently, this compound was shown to induce apoptosis in a variety of cancer cells with promising in vivo antitumor activity in canine lymphoma model. Recently, we have reported its ability to kill drugresistant, $\mathrm{Bcl}-2 / \mathrm{Bcl}-\mathrm{xL}$ overexpressing and Bax/Bak-deficient cells despite the essential requirement of mitochondrial cytochrome $c$ (cyt. c) release for caspase activation, indicating that the key molecular targets of PAC-1 in cancer cells are yet to be identified. Here, we have identified Ero1 $\alpha$-dependent endoplasmic reticulum (ER) calcium leakage to mitochondria through mitochondria-associated ER membranes (MAM) and ER luminal hyper-oxidation as the critical events of PAC-1-mediated cell death. PAC-1 treatment upregulated Ero1 $\alpha$ in multiple cell lines, whereas silencing of Ero1 $\alpha$ significantly inhibited calcium release from ER and cell death. Loss of ER calcium and hyper-oxidation of ER lumen by Ero1 $\alpha$ collectively triggered ER stress. Upregulation of GRP78 and splicing of X-box-binding protein 1 (XBP1) mRNA in multiple cancer cells suggested ER stress as the general event triggered by PAC-1. XBP1 mRNA splicing and GRP78 upregulation confirmed ER stress even in Bax/Bak double knockout and PAC-1-resistant Apaf-1-knockout cells, indicating an induction of ER stress-mediated mitochondrial apoptosis by PAC-1. Furthermore, we identified BH3-only protein p53 upregulated modulator of apoptosis (PUMA) as the key molecular link that orchestrates overwhelmed ER stress to mitochondria-mediated apoptosis, involving mitochondrial reactive oxygen species, in a p53-independent manner. Silencing of PUMA in cancer cells effectively reduced cyt. $c$ release and cell death by PAC-1.

Cell Death and Disease (2013) 4, e968; doi:10.1038/cddis.2013.502; published online 19 December 2013

Subject Category: Cancer

Caspases existing as zymogens inside the cell act as key players in initiation and execution of apoptosis, after their activation by proteolysis. Death signals induced by anticancer drugs are transmitted from the mitochondria to downstream executioner caspases such as caspase- $3,-7$ and -6 and it is believed that cells succumb to death due to cleavage of several substrate proteins by activated forms of these executioner caspases. ${ }^{1,2}$ However, a subset of cancers are not responsive to anticancer drugs owing to defective apoptotic machinery. ${ }^{3,4}$ In general, drug-resistant cancer cells show defects in apoptosis upstream of caspase activation, at the level of mitochondrial membrane permeabilization. ${ }^{5}$ Hence, identification of small-molecular compounds that can directly activate procaspase- 3 downstream of mitochondria is a promising avenue for targeting clinical drug-resistance. ${ }^{6,7}$

Procaspase-activating compound-1 (PAC-1) is the first small compound reported to convert procaspase-3 to active caspase-3, in vitro, by chelating inhibitory zinc ions from procaspase-3, thereby inducing effective cell death in tumor cells as well as mouse xenograft models. ${ }^{8,9}$ This compound recently received increased attention because of its selective activity on cancer cells and promising antitumor activity in the canine lymphoma model. ${ }^{10}$ Our recent report, where in which we have used several experimental cellular model systems to define direct caspase activation, demonstrated that PAC-1 essentially requires cytochrome $c$ (cyt. $c$ ) release from the mitochondria and Apaf-1 to induce caspase activation. ${ }^{11}$ Moreover, the study identified the potential activity of PAC-1 in triggering cyt. $c$ release in a Bax/Bak-independent manner and in bypassing drug-resistance mediated by Bcl-2 family proteins. In vivo antitumor activity as well as bio-availability studies place PAC-1 as a strong candidate antitumor agent; furthermore, preclinical studies of PAC-1 derivatives are ongoing in other tumor models. ${ }^{10,12}$

\footnotetext{
${ }^{1}$ Cancer Research Program-1, Rajiv Gandhi Centre for Biotechnology, Thiruvananthapuram, Kerala, India

*Corresponding author: TR Santhoshkumar, Cancer Research Program-1, Rajiv Gandhi Centre for Biotechnology, Thycaud P.O., Poojappura, Thiruvananthapuram, Kerala 695 014, India. Tel: +91 471 2341716; Fax: +91 471 2348096; E-mail: trsanthosh@rgcb.res.in

Keywords: ER stress; PAC-1; Ero1 $\alpha$; ER calcium; procaspase activation; apoptosis

Abbreviations: UPR, unfolded protein response; Cyt. c cytochrome $c ; \Delta \Psi_{\mathrm{m}}$, mitochondrial membrane potential; DKO, double knockout; ER, endoplasmic reticulum; Ero1 $\alpha$, endoplasmic reticulum oxidoreductin-1 alpha; FRET, fluorescence resonance energy transfer; GFP, green fluorescent protein; PAC-1, procaspase-activating compound-1; PI, propidium iodide; PUMA, p53 upregulated modulator of apoptosis; KO, knockout; MAM, mitochondria-associated ER membrane; ROS, reactive oxygen species; WT, wild-type

Received 25.8.13; accepted 10.10.13; Edited by A Finazzi-Agró
} 
Despite their promising antitumor activity, cell death mechanism and important cellular targets of these compounds in cancer cells are yet to be identified.

In this report, we have delineated the cell death mechanism induced by PAC-1. The study suggests that, PAC-1-induced mitochondrial permeabilization is mediated through mitochondrial calcium overload and mitochondrial reactive oxygen species (ROS), assisted by endoplasmic reticulum (ER) oxidoreductin-1 alpha (Ero1 $\alpha$ )-dependent calcium release from the ER. Our study also revealed p53-independent upregulation of p53 upregulated modulator of apoptosis (PUMA) that assists mitochondrial permeabilization and acts as a molecular link between ER and mitochondria during cell death.

\section{Results}

PAC-1 induces apoptotic cell death, G1 arrest and autophagy in multiple cancer cell lines even in the absence of caspase-3. Multiple cancer cell lines were used to study apoptosis and cell cycle arrest induced by PAC-1. Chromatin condensation and propidium iodide (PI) staining data (Figure 1a and Supplementary Figure S1a) indicate that SKOV3, U2OS and ADR-RES cells are relatively resistant to PAC-1 than other cell types used. Consistent with our previous report, MCF7 cells remained sensitive to PAC-1 in comparison with other cell types, despite being deficient for caspase-3. ${ }^{11}$ Significant annexin $\mathrm{V}$ binding was noticed in MCF7 cells and re-expression of caspase-3 in MCF7 cells enhanced apoptosis (Figures $1 \mathrm{~b}$ and $\mathrm{c}$ ). Analysis of cell cycle at early time point $(12 \mathrm{~h})$ indicates that PAC-1 treatment induced $G_{1}$ arrest before triggering cell death (Figure 1d). The most common signaling that contributes to cell cycle arrest and cell death is DNA damage response. ${ }^{13}$ Therefore, we examined whether PAC-1 induces DNA damage, using phospho-H2AX as DNA damage indicator, in HeLa cell line (Figure 1e). PAC-1 treatment failed to induce DNA damage response in HeLa cells, which suggests that PAC- 1 induces cell death not by directly damaging DNA. In order to analyze whether PAC-1 induces autophagy, another form of cell death, we studied autophagy marker microtubuleassociated protein 1A/1B-light chain 3 (LC3B) expression both in the presence and absence of caspase- 3 due to its association with autophagic vesicles. ${ }^{14}$ Interestingly, aggregation of LC3 was observed in both cell lines (Figure 1f) upon PAC-1 treatment. Furthermore, the conversion of LC3-I and LC3-II was also observed in western blot (Figure 1g). Time-lapse imaging employing MCF7 cells expressing EGFP-LC3 showed time-dependent LC3 aggregation associated with autophagosome formation (Supplementary Video S1). PAC-1 also induced autophagy in mouse embrogenic fibroblast (MEF) Bax/ Bak double knockout (DKO) cells (Supplementary Figure $\mathrm{S} 1 \mathrm{~b})$. Even though the results substantiated existence of autophagy in PAC-1-treated cells, it is unclear whether cells use this 'self digestion' as a means of survival or death. However, autophagy inhibition could not inhibit PAC-1-induced cell death but enhanced it (Figure 1h), indicating that PAC-1-mediated autophagy is a stress adaptation and does not contribute to cell death.
Induction of UPR and ER stress-regulated proteins in cancer cell lines as well as MEF Apaf-1 KO and Bax/Bak DKO cell lines. Chemotherapy-induced apoptosis is orchestrated by the engagement of multiple pro-apoptotic and anti-apoptotic survival proteins. However, western blot analysis of key proteins indicated no variation in expression of Bcl-2, Bak, Bax, XIAP, Hsp90, Hsp27 and Hsp70 in PAC-1-treated cells against untreated cells (Figure 2a). Surprisingly, upregulation of ER-chaperons GRP78 and 94 was noticed in both MCF7 and MCF7C3 cells, indicating induction of unfolded protein response (UPR) that occurs in response to ER stress. To further characterize ER stress, various regulators of ER stress such as p-elF $2 \alpha, \mathrm{CHOP}$, inositol requiring kinase $\alpha(\operatorname{IRE} 1 \alpha)$, Ero $1 \alpha$ and calnexin were analyzed by western blot (Figure 2b). PAC-1 treatment induced upregulation of CHOP, p-elF $2 \alpha, \operatorname{IRE} 1 \alpha$ and Ero $1 \alpha$, supporting its ability to trigger ER stress independent of caspase-3. GRP78 was upregulated in multiple cancer cell lines upon PAC-1 treatment (Figure 2c), including PAC-1resistant cells such as SKOV3 and U2OS. Upregulation of GRP78 was also noticed in Bak/Bax DKO cells and Apaf-1-knockout (KO) cells (Figures $2 e$ and f). Upon accumulation of misfolded proteins in ER, BiP/GRP78 gets released and allows oligomerization of IRE $1 \alpha$ and protein kinase-like ER kinase (PERK), activating both. Activated IRE $1 \alpha$ disposes an intrinsic endoribonuclease activity that mediates the unconventional splicing of $\mathrm{X}$-box-binding protein 1 (XBP1) mRNA. ${ }^{15}$ Splicing of XBP1 mRNA was noticed in all cells after PAC-1 treatment, including Apaf-1and Bax/Bak-deficient cells (Figures $2 \mathrm{~d}-\mathrm{f}$ ). Apaf- 1 deficiency prevented PAC-1-mediated cell death but not XBP1 splicing, substantiating the requirement of Apaf-1 in ER stress-mediated apoptosis.

GRP78-silencing enhances and cycloheximide treatment reduces PAC-1-mediated cell death. Proper folding, maturation and stabilization of nascent proteins in ER are assisted and monitored by several resident chaperons such as GRP78 and GRP94. During UPR, these chaperons get upregulated as a survival mechanism to recover cells from ER stress. It was previously reported that silencing of GRP78 sensitizes cells to ER stress-mediated apoptosis. ${ }^{16,17}$ Therefore, cell death was analyzed in GRP78-silenced MCF7 and HeLa cells by PI staining using flow cytometry (Figures $3 a$ and b). As shown, PAC-1 induced more cell death in GRP78-silenced cells. Inhibition of protein translation with cycloheximide $(2 \mu \mathrm{g} / \mathrm{ml})$ can ameliorate effects of ER stress by decreasing protein burden on ER and can reduce ER stress-mediated apoptosis. ${ }^{15}$ Therefore, MCF7 and HeLa cells expressing caspase-cleavable, specific fluorescence resonance energy transfer (FRET) probe, CFP-DEVD-YFP, were treated with PAC-1 in the presence and absence of cycloheximide. As shown in Figure $3 c$, cycloheximide treatment reduced number of cells with caspase activation compared with PAC-1 treatment alone. Furthermore, we evaluated cell death in PAC-1-sensitive HCT116 and MCF7 cells both with and without cycloheximide treatment by $\mathrm{PI}$ staining and clonogenicity assay (Figures $3 d$ and e), which again substantiated the reduction in cell death upon cycloheximide pretreatment. The results 


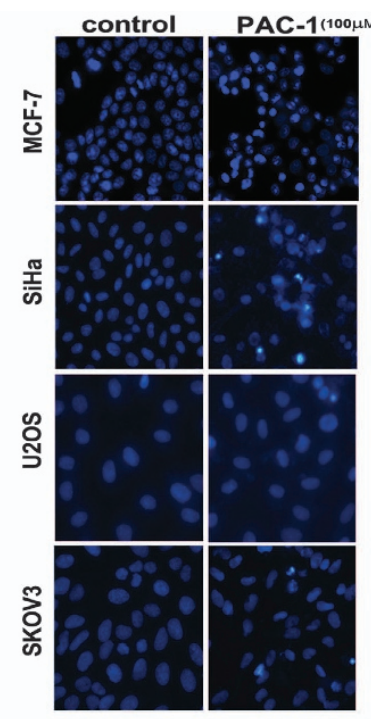

b

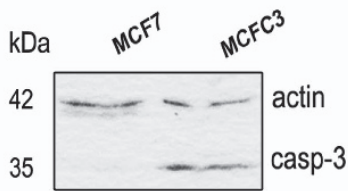

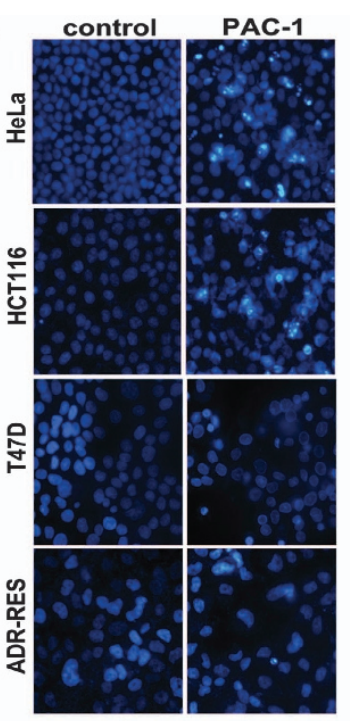

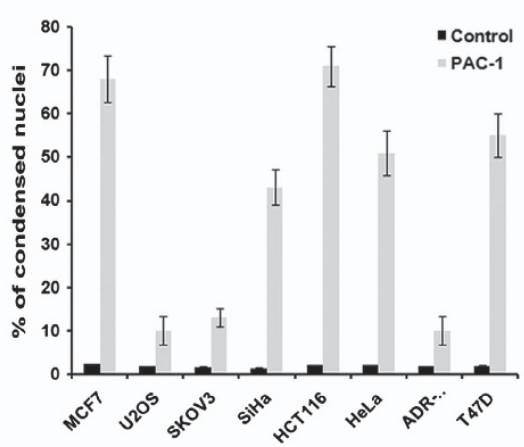

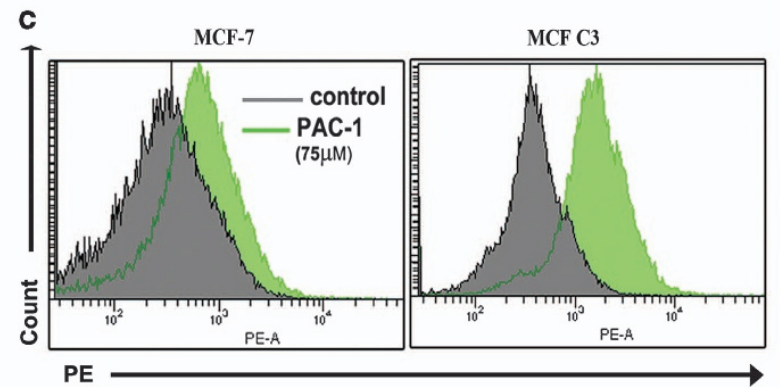

d

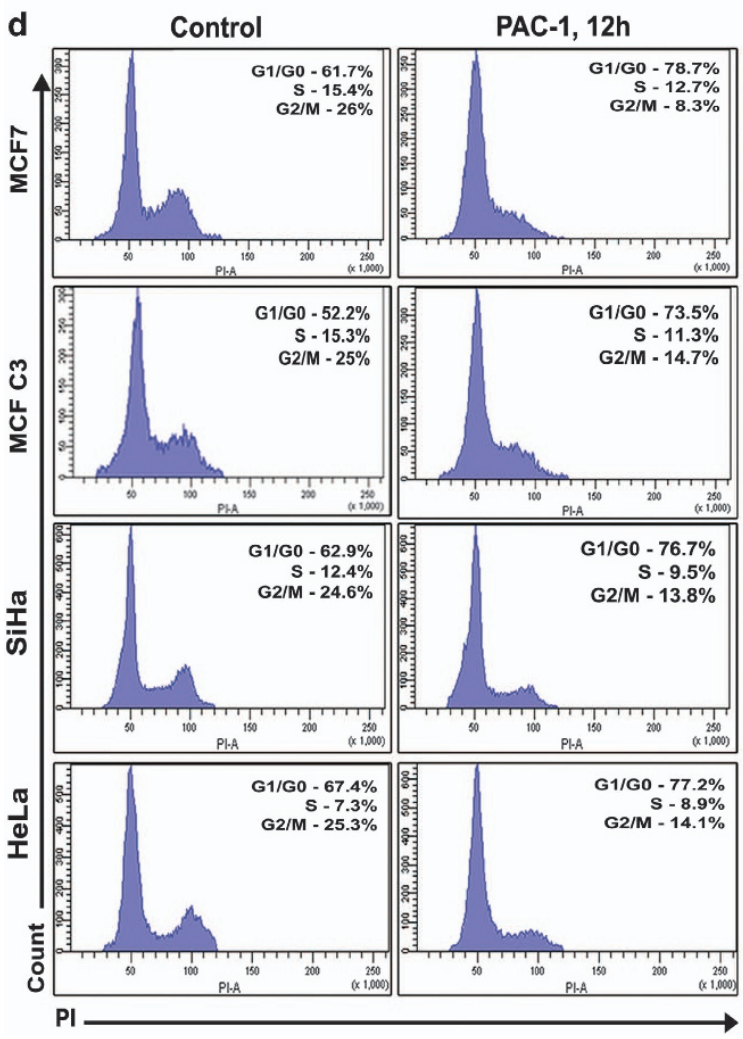

e

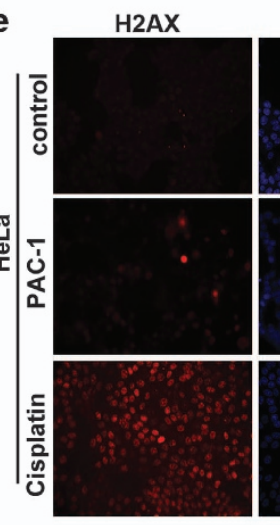

Nucleus

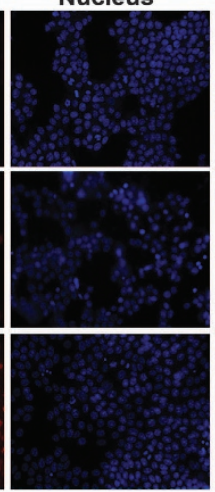

merged

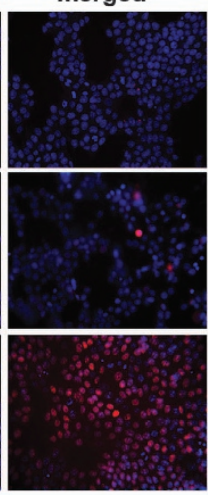

Figure 1 (Continued) 
f
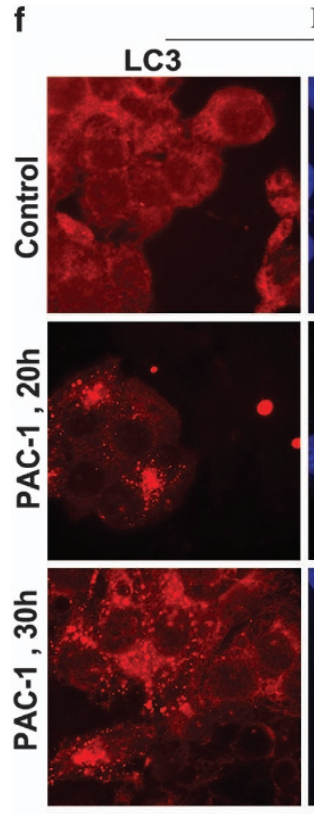

MCF7
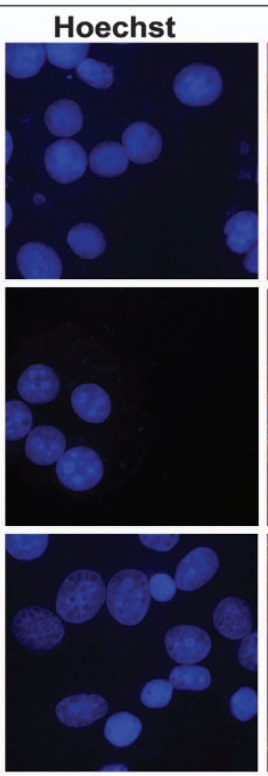

\section{Merged}
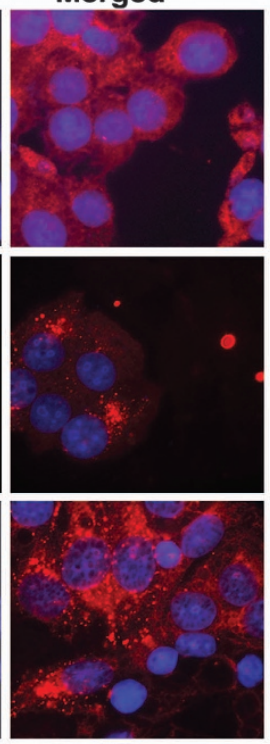

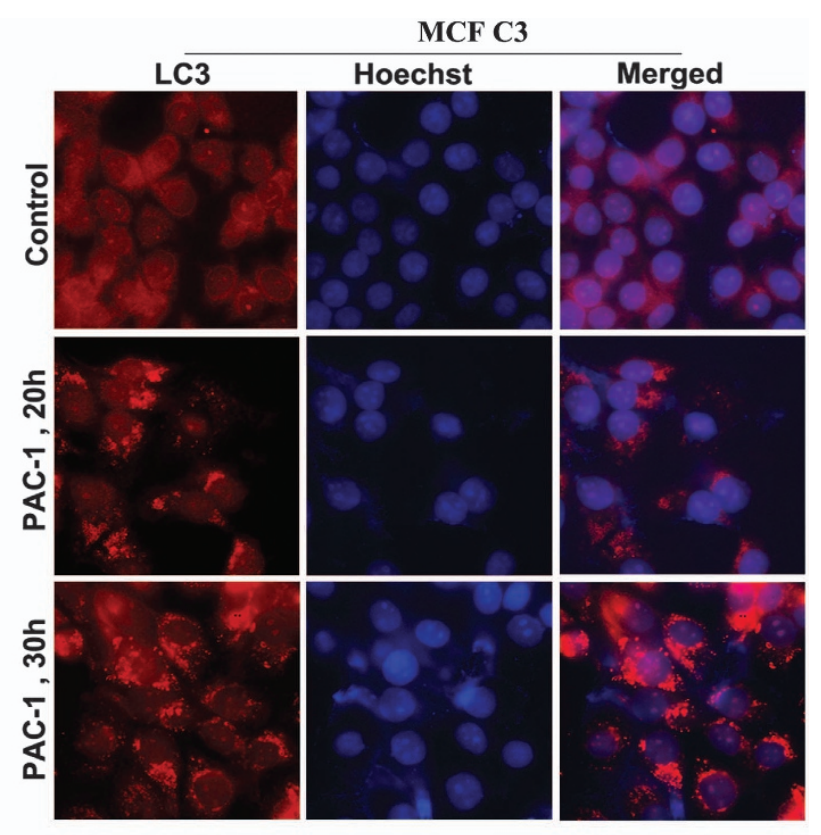

g

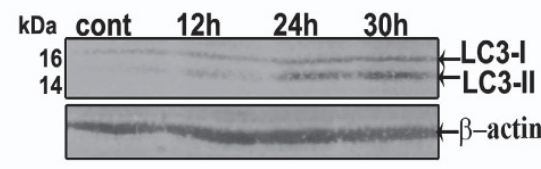

h

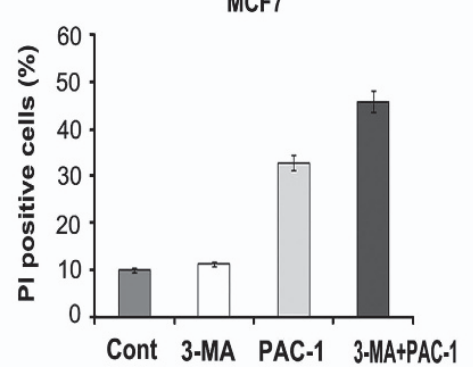

MCF C3

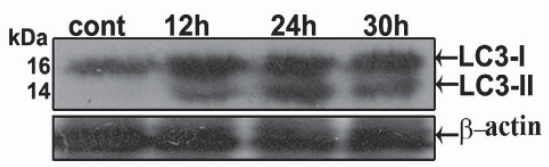

MCF C3

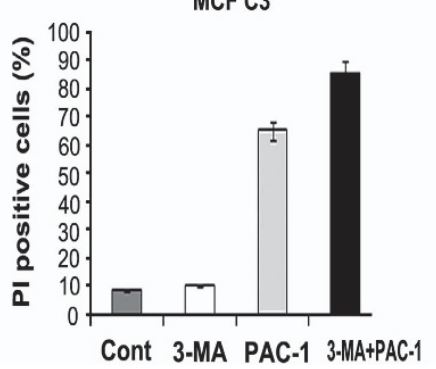

Figure 1 PAC-1 induces G1 arrest, cell death and autophagy irrespective of caspase-3 expression and without signs of DNA damage. (a) PAC-1 induced chromatin condensation in multiple cancer cell lines such as HCT116, SiHa, HeLa, SKOV3, ADR-RES, T47D, U2OS, T47D and caspase-3-deficient MCF7 cells. The percentages of condensed nuclei are represented graphically $(n=3$, mean \pm S.D.). (b) Western blot showing the expression of stably transfected procaspase-3 in MCF7 cells lacking caspase-3. (c) FACS analysis of apoptosis in MCF7 and MCF7C3 by annexin V staining after PAC-1 $(75 \mu \mathrm{M}, 24 \mathrm{~h})$ treatment. (d) PAC-1 (75 $\mu \mathrm{M}, 12 \mathrm{~h})$ arrested MCF7, MCF7C3, SiHa and HeLa cells in $G_{1}$ phase of cell cycle before inducing apoptosis. (e) Immunofluorescence staining using H2AX antibody in HeLa cells showed that PAC-1 (100 $\mu$ M, $24 \mathrm{~h}$ ) did not induce any DNA damage. Cisplatin was used as positive control, which induced DNA damage, evident from the enhanced red fluorescence observed than untreated cells. (f) Immunofluorescence detection of LC3 suggested that PAC-1 induced massive autophagic granularization of LC3 in both MCF7 and MCF7C3 cells. (g) PAC-1-induced enhanced expression of LC3-II protein was detected by western blot in both MCF7 and MCF7C3 cells. $\beta$-Actin was used as loading control. (h) Addition of autophagy inhibitor 3-methyladenine (3-MA; $5 \mathrm{mM}$ ) along with PAC-1 could not inhibit cell death. Cell death was analyzed by PI staining using FACS and percentage of PI-positive cells are represented graphically for both MCF7 and MCF7C3 cells $(n=3$, mean \pm S.D.)

described above imply that PAC-1 induces ER stressmediated apoptosis, which can be reduced by ER stress inhibitors.

Anti-apoptotic Bcl-2 family proteins exert their anti-apoptotic activity by inhibiting the release of cyt. $c$ from the mitochondria irrespective of its spatial localization at ER or mitochondria. ${ }^{18}$ We have previously reported that PAC-1 can bypass Bcl-2 and $\mathrm{Bcl}-\mathrm{xL}$-mediated drug-resistance. ${ }^{11}$ However, several studies propose that ER-associated $\mathrm{Bcl}-2$ protein is capable of inhibiting apoptosis induced specifically by ER stressinducing agents such as thapsigargin and tunicamycin. ${ }^{19,20}$ Therefore, we generated MCF7C3 cells transfected stably with Bcl-2Cb5-EGFP plasmid (Supplementary Figure S2a). Evaluation of cell death by nuclear condensation suggested that ER-targeted Bcl-2 inhibited nuclear condensation significantly upon PAC-1 treatment (Supplementary Figure S2b). It also delayed cyt. $c$ release and caspase activation (Supplementary Figures S2c-e). These results hint that 
$\mathrm{Bcl}-2$ associated with ER can confer resistance against PAC-1mediated apoptosis, which proceeds via the mitochondria as reported in our previous report. ${ }^{21}$

PUMA-mediated mitochondrial permeabilization links ER stress and mitochondrial death signaling. Tumorsuppressor p53 has major role in coordinating multiple cellular processes such as cell cycle, apoptosis and ER stress-mediated apoptosis by induction of pro-apoptotic Bcl2 family proteins PUMA and NOXA. ${ }^{15,22}$ To unravel the importance of p53 in PAC-1-induced cell death, the status of p53 was evaluated in multiple cancer cell lines after PAC-1 treatment, which proved its ability to upregulate p53 (Figure 4a). However, PAC-1 induced cell death even in p53 KO HCT116 cells, although it was partially suppressed than in its wild-type (WT) counterpart as concluded from nuclear condensation and clonogenicity assays (Figures 4b and c), signifying p53-independent death signaling. Mitochondrial permeabilization during cell death is primarily regulated by evolutionarily conserved $\mathrm{Bcl}-2$ family proteins. Proteins of BH3-only subgroup of Bcl-2 family proteins such as PUMA, Bim and Bik are the key factors that transfer death signals from ER to mitochondria during ER stress. ${ }^{23,24}$ Therefore, levels of these proteins were assessed in multiple cancer cell lines such as MCF7, SiHa and HCT116 after PAC-1 treatment (Figure 4d). Even though Bim and Bik levels varied in a cell-dependent manner after PAC-1 treatment, PUMA was found upregulated in all the cell lines consistently. PUMA is known to get transactivated by p53 during genotoxic stress. ${ }^{25}$ PUMA is also activated by other transcription factors to initiate p53-independent apoptotic responses to nongenotoxic stimuli, including growth factor/ cytokine deprivation, ER stress and ischemia/reperfusion. ${ }^{25}$

As PAC-1 killed p53 $\mathrm{KO}$ cells in spite of its ability to upregulate p53 in multiple cancer cell lines, we studied the role of p53 in PUMA upregulation. Surprisingly, PUMA was highly upregulated even in the absence of p53 (Figure 4d), indicative of PAC-1-induced p53-independent upregulation of PUMA in various cancer cell lines, which seems to be the common molecular link between ER stress and mitochondrial apoptotic pathway. To further confirm the essential role of PUMA in mediating mitochondrial permeabilization and cyt. $c$ release, MCF7 cells stably expressing cyt. $c$-EGFP were transfected with either shRNA against PUMA or control vector followed by PAC-1 treatment. Silencing of PUMA in cyt. $c$-EGFP-expressing cells reduced both cyt. $c$ release and cell death induced by PAC-1 (Figures $4 \mathrm{e}-\mathrm{g}$ and Supplementary Figure S3), confirming the crucial role of PUMA in mediating ER stress-dependent cell death by PAC-1.

Ero1 $\alpha$-mediated calcium flux from ER to mitochondria through engagement of MAM. The results described so far suggest for a role of ER stress and PUMA-dependent mitochondrial permeabilization in PAC-1-induced cell death. ER is not only the major site for protein folding, maturation and stabilization, it also has a crucial role in calcium homeostasis. ${ }^{26,27}$ Therefore, to dissect the role of $\mathrm{Ca}^{2+}$ in PAC-1-induced ER stress, HeLa cells were stably transfected with calcium sensor, D1ER cameleon, to detect ER calcium release. ${ }^{28}$ PAC-1 triggered significant change in
ECFP/EYFP ratio, representing the release of calcium from ER by PAC-1 (Figure 5a). Role for Bik and Bim in ER calcium release has been reported in multiple forms of ER stressinduced cell death. ${ }^{29,30}$ However, the induction of Bik and Bim was not significant and consistent in PAC-1-treated cells, ruling out their role in mediating ER calcium release. Recent studies have implicated the role of CHOP and Ero1 $\alpha$ in the regulation of ER calcium. ${ }^{31,32}$ As these two proteins were highly upregulated in PAC-1-treated cells as described earlier, to confirm their role in ER calcium release, both Ero1 $\alpha$ and $\mathrm{CHOP}$ were silenced in HeLa D1ER cells (Figures 5bd). Silencing of Ero1 $\alpha$ reduced ER calcium release, as reflected from lesser FRET-ratio change (Figure 5c) along with reduced cell death (Figure $5 \mathrm{~d}$ ) observed in Ero1 $\alpha$ silenced cells than in CHOP-silenced cells.

The above studies indicate that Ero1 $\alpha$ is a critical component of ER calcium release and subsequent cell death by PAC-1. The increased cell death inhibition found in Ero1 $\alpha$-silenced cells than in CHOP-silenced cells suggests for an Ero1 $\alpha$-dependent dominant cell death signaling in PAC-1-treated cells. Therefore, the essential role of Ero1 $\alpha$ in PAC-1-induced cell death was further analyzed in a panel of cancer cell lines. As shown in Figure $5 e$, an early induction of Ero1 $\alpha$ was evident in all cells, even in p53 KO HCT116 cells and Bax/Bak DKO MEF cells. Ero1 $\alpha$ is a key component of ER-mitochondrial membrane that is important for the ER-mitochondrial calcium signaling. ${ }^{33-35}$ Ero1 $\alpha$ upregulation may allow the formation of mitochondria-associated ER membrane (MAM), leading to mitochondrial calcium uptake resulting in mitochondrial permeabilization. So as to visualize MAM formation by PAC-1, we generated cells expressing EGFP targeted at ER and DsRed fluorescent protein targeted at mitochondria. Both high resolution microscopic images and confocal imaging revealed structural changes of both ER and mitochondria with occasional close association points, in PAC-1-treated cells compared with untreated cells (Figure $5 f$ and Supplementary Figure S4). As evident from microscopic images, PAC-1-treated cells showed more globular ER compared with the network structures in untreated cells, demonstrating that profound morphological change may assist MAM formation in PAC-1-treated cells. Overall, the results described above strongly suggest that PAC-1-induced Ero1 $\alpha$-upregulation culminates in ER calcium leakage specifically into the mitochondria through engagement of MAM.

ER hyper-oxidation leads to mitochondrial calcium uptake and mitochondrial ROS generation. MAM-dependent calcium release from the ER primarily allows selective calcium uptake by mitochondria. Therefore, we analyzed mitochondrial calcium uptake in both HeLa and MEF Bax/Bak DKO cells using mitochondrial calcium indicator dye, Rhod-2, by flow cytometry, that showed a PAC-1stimulated increased uptake of mitochondrial calcium (Figure 6a). Consistent with this, pretreatment of cells with either mitochondrial calcium uptake inhibitor Ru360 or BAPTA-AM prevented cell death induced by PAC-1 both in HeLa and MEF Bax/Bak DKO cells (Figure 6b). A possible mechanism of mitochondrial calcium overload-dependent mitochondrial permeabilization is through generation of mitochondrial ROS. To study the generation of mitochondrial 
6
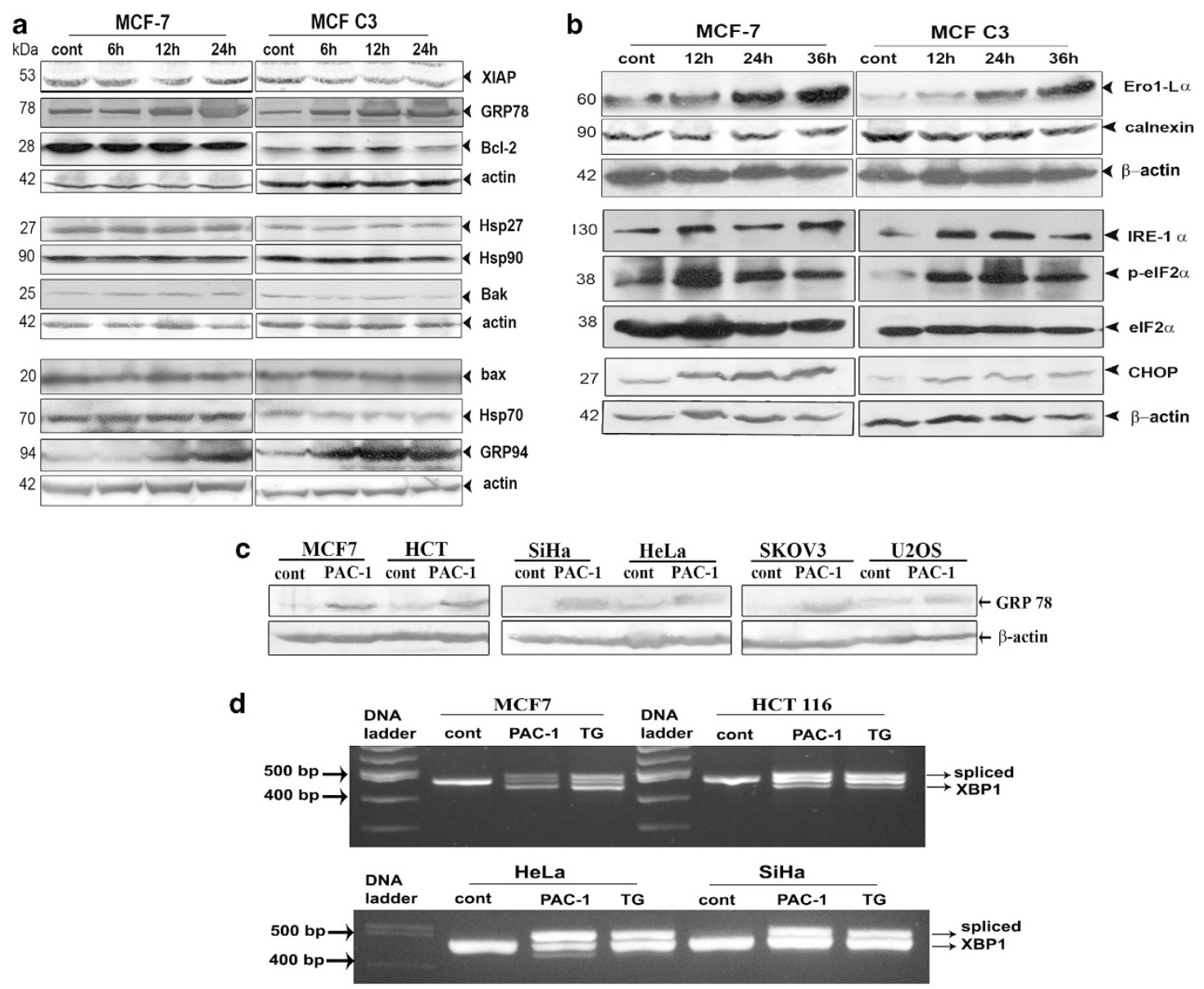

e
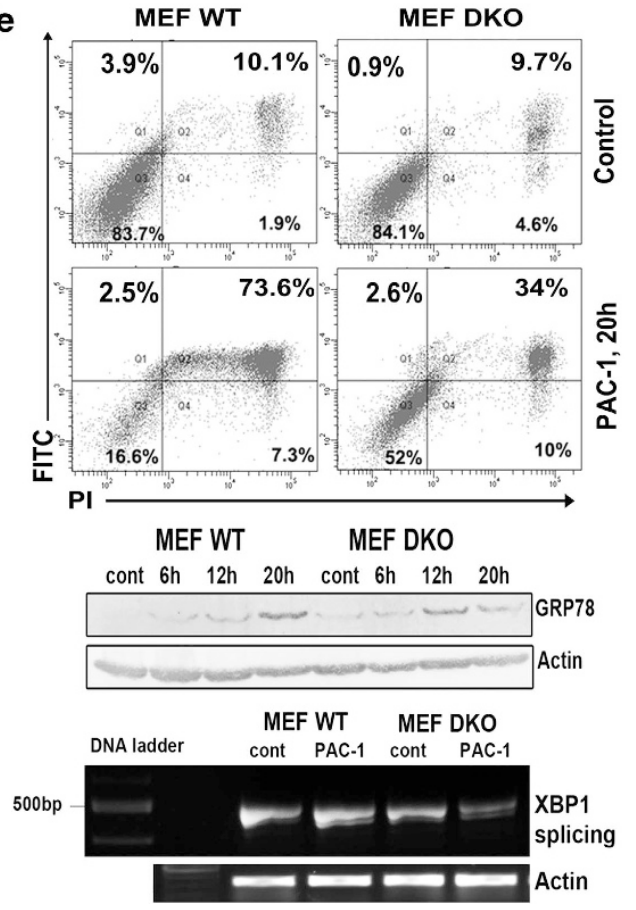

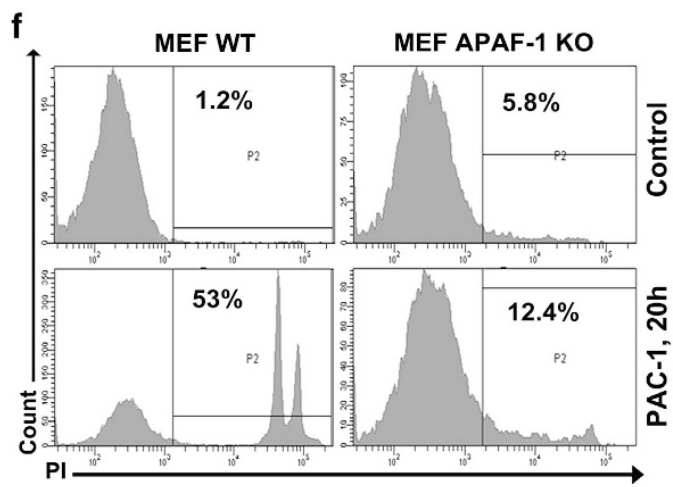

MEF WT MEF Apaf-1 KO

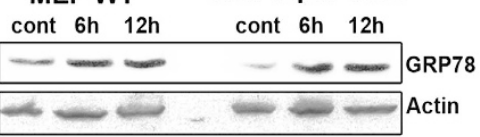
DNA MEF WT MEF Apaf-1 KO ladder $0 \mathrm{~h} \quad 12 \mathrm{~h} \quad 20 \mathrm{~h}$ oh $12 \mathrm{~h} \quad 20 \mathrm{~h}$

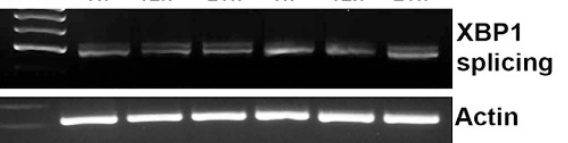


ROS by PAC-1, HeLa cells were stably expressed with redox sensitive green fluorescent protein (GFP) targeted at the mitochondria, roGFP-Mito. ${ }^{36}$ The change in excitation ratio at 405 and $488 \mathrm{~nm}$ reflects the oxidized or reduced state of the probe at the mitochondria. Ratio imaging by microscopy revealed an increase in $405 / 488 \mathrm{~nm}$ ratio in more than $32 \%$ of cells after $12 \mathrm{~h}$ of PAC-1 treatment, specifying oxidation of the probe (Figure 6c). As shown in supplementary Figure S5, $46 \%$ of cells showed increase in $405 / 488 \mathrm{~nm}$ ratio after PAC1 treatment, substantiating the oxidation of roGFP-Mito as obvious from flow cytometry. DTT completely reduced the mitochondrial probe with a reduction in $405 / 488 \mathrm{~nm}$ ratio compared with untreated cells, indicating the functionality of the sensor. As Ero1 $\alpha$ is capable of oxidizing ER lumen that culminates in ER calcium release, we suspected that PAC-1 may hyper-oxidize ER lumen that initiates ER calcium release. To generate evidence for the hyper-oxidation of ER by PAC-1, HeLa cells were transfected with redox sensitive GFP targeted at ER, roGFP2-iL-KDEL as described recently. ${ }^{37}$ The ratio imaging as well as flow cytometry analysis revealed oxidation of roGFP targeted at the ER, demonstrating that ER luminal oxidation is indeed triggered by PAC-1, possibly through Ero1 $\alpha$ (Figure $6 \mathrm{~d}$ and Supplementary Figure 5).

\section{Discussion}

Compounds that can bypass the anti-apoptotic function of $\mathrm{Bcl}-$ 2/Bcl-xL and trigger mitochondrial cyt. $c$ release independent of Bax/Bak may constitute a therapeutic goal against drugresistant cancers. ${ }^{3}$ In this approach, direct activation of executioner caspases remains as a promising strategy, as activated caspases can kill cells quickly irrespective of the expression of prominent anti-apoptotic proteins. ${ }^{6,7}$ The first identified direct procaspase-3 activator (PAC-1), even though failed to activate caspases directly in cells, showed the capability to kill drug-resistant Bax/Bak-deficient cells as well as Bcl-2 overexpressing cancer cells as accounted in our previous report. ${ }^{8,11}$ Moreover, promising bio-availability and in vivo antitumor activity establishes PAC-1 as a potential anticancer drug. ${ }^{10}$ In this study, we report that PAC-1 triggers ER stress signaling subsequent to Ero1 $\alpha$-dependent ER luminal hyper-oxidation leading to calcium leakage with simultaneous induction of autophagy and mitochondriamediated apoptosis. A recent limited microarray-analysis also implicated possible ER stress signaling in PAC-1-induced cell death. $^{38}$
The accumulation of unfolded proteins in ER represents a cellular stress induced by multiple stimuli as well as pathological conditions, which triggers evolutionarily conserved adaptive response termed UPR and leads to cell death if it is severe or protracted. ${ }^{15}$ PAC-1 induced UPR in multiple cancer cell lines irrespective of caspase-3 status as indicated by early upregulation of ER-chaperons such as GRP78 and GRP94. When unfolded proteins accumulate in the ER, ER transmembrane sensors detect them to initiate three distinct UPR branches mediated by protein molecules such as PERK, IRE $1 \alpha$ and transcription factor ATF6 to deal with accumulated unfolded proteins. ${ }^{15}$ Phosphorylation of elF $2 \alpha$ and upregulation of CHOP was noticed in both MCF7 and MCF7C3 cells upon PAC-1 treatment, suggestive of the activation of PERK and ATF4/ATF6 branches of UPR signaling. IRE $1 \alpha$ is the most fundamental of ER stress sensors, which is conserved in all eukaryotic cells and activation of which elicits endoribonuclease activity that specifically cleaves mRNA encoding transcriptional factor XBP1. ${ }^{15,22}$ Consistent with this, unconventional splicing of XBP1 was observed in several cancer cell lines upon PAC-1 treatment. Moreover, PAC-1 induced GRP78 upregulation and XBP1 splicing in Bax/Bak DKO and Apaf-1 KO cells. Interestingly, the absence of Apaf-1 inhibited PAC-1-mediated cell death but not UPR response, proving that PAC-1-mediated ER stress induction precedes the initiation of mitochondrial death signaling. Translational inhibitor cycloheximide and overexpression of ER-targeted $\mathrm{Bcl}-2$ significantly reduced caspase activation and consequent cell death, indicating that PAC-1-induced ER stress transduces death signal from the ER to mitochondria that causes cyt. $c$ release and subsequent caspase activation. Although PAC-1 induced autophagy, inhibition of autophagy enhanced cell death, suggesting it as an initial survival signaling in PAC-1-treated cells. UPR is tailored essentially to re-establish ER homeostasis also through adaptive mechanisms involving stimulation of autophagy. ${ }^{39,40}$

The physical contact point between ER and mitochondria, which acts as a signaling hub, called MAM, is enriched with several proteins that allow integration of signaling between these two organelles. Ero1 $\alpha$ induction by PAC-1 may allow formation of microdomains of calcium channels, leading to selective uptake of calcium into mitochondria through mitochondrial $\mathrm{Ca}^{2+}$ uniporter (MUC). This chronic leakage of calcium to mitochondria culminates in mitochondrial calcium overload as well as ER luminal calcium deficiency. Previous studies have shown that ER stress contributes to CHOP-dependent induction of Ero1 $\alpha .{ }^{31}$ We have observed

Figure 2 PAC-1 induces UPR and ER stress in multiple cell types including apoptotic defective cell lines. (a) Western blot demonstrates that PAC-1 did not alter the expression of proteins XIAP, Bak, Bak, Bcl-2, Hsp27, Hsp70 and Hsp90 significantly but it could induce significant upregulation of ER stress marker GRP78 and GRP94 proteins in both MCF7 and MCF7C3 cells. $\beta$-Actin was used as loading control. (b) PAC- 1 induced expression of several UPR indicator proteins such as CHOP, IRE1 $\alpha$, Ero1 $\alpha$ and p-elF2 $\alpha$ both in MCF7 and MCF7C3 cells. $\beta$-Actin was used as loading control. (c) PAC-1 treatment upregulated ER stress marker protein GRP78 in multiple cancer cell lines such as MCF7, HCT116, U2OS, SKOV3, SiHa and HeLa. $\beta$-Actin was used as loading control. (d) Splicing of XBP1 mRNA was observed by RT-PCR in multiple cancer cells such as MCF7, HCT116, SiHa and Hela after PAC-1 treatment. Thapsigargin was used as positive control. (e) Cell death was analyzed by annexin V binding and PI staining in MEF WT and Bax/Bak DKO cells using FACS. The percentages of PI and annexin-positive cells are mentioned in Q2 quadrant representing apoptotic cells. Western blot and RT-PCR results suggest that PAC-1 induced upregulation of GRP78 as well as splicing of XBP1 mRNA in both MEF WT and DKO cells. $\beta$-Actin was used as loading control. (f) Cell death was analyzed by PI staining in MEF WT and MEF Apaf-1 KO cells using FACS after PAC- 1 (50 $\mu$ M) treatment. There was negligible cell death in Apaf-1 $\mathrm{KO}$ cells than its WT counterpart. The percentage of PI-positive cells is represented in P2 gate. Furthermore, western blot and RT-PCR results suggest that PAC-1 induced splicing of XBP1 mRNA and upregulation of GRP78 efficiently in WT as well as Apaf-1 KO cells even though cell death was absent in Apaf-1 KO cells 
a

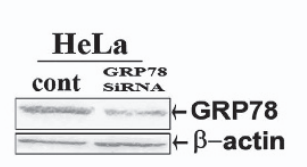

b
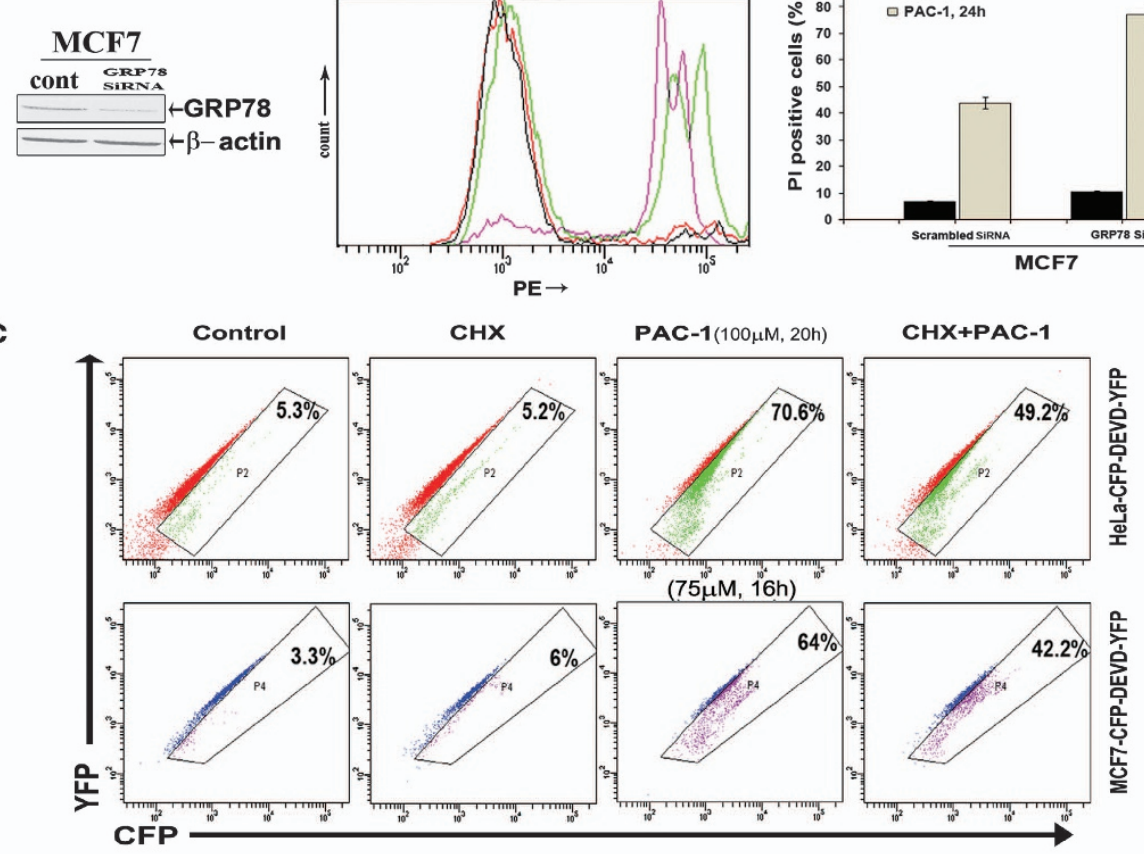

HeLa
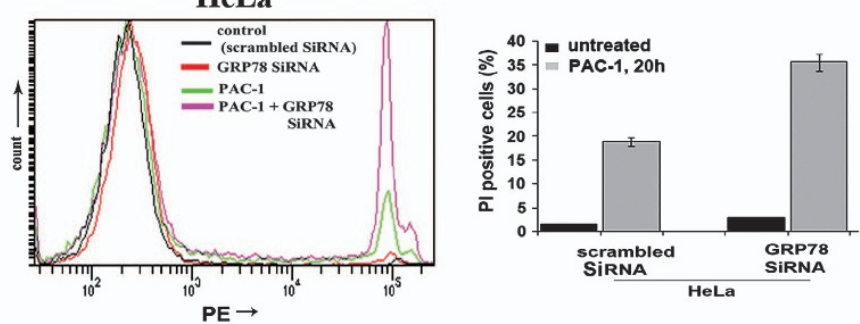

MCF7
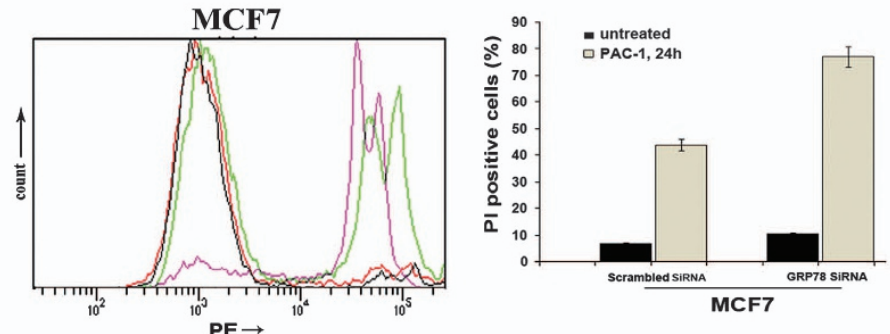

C

e
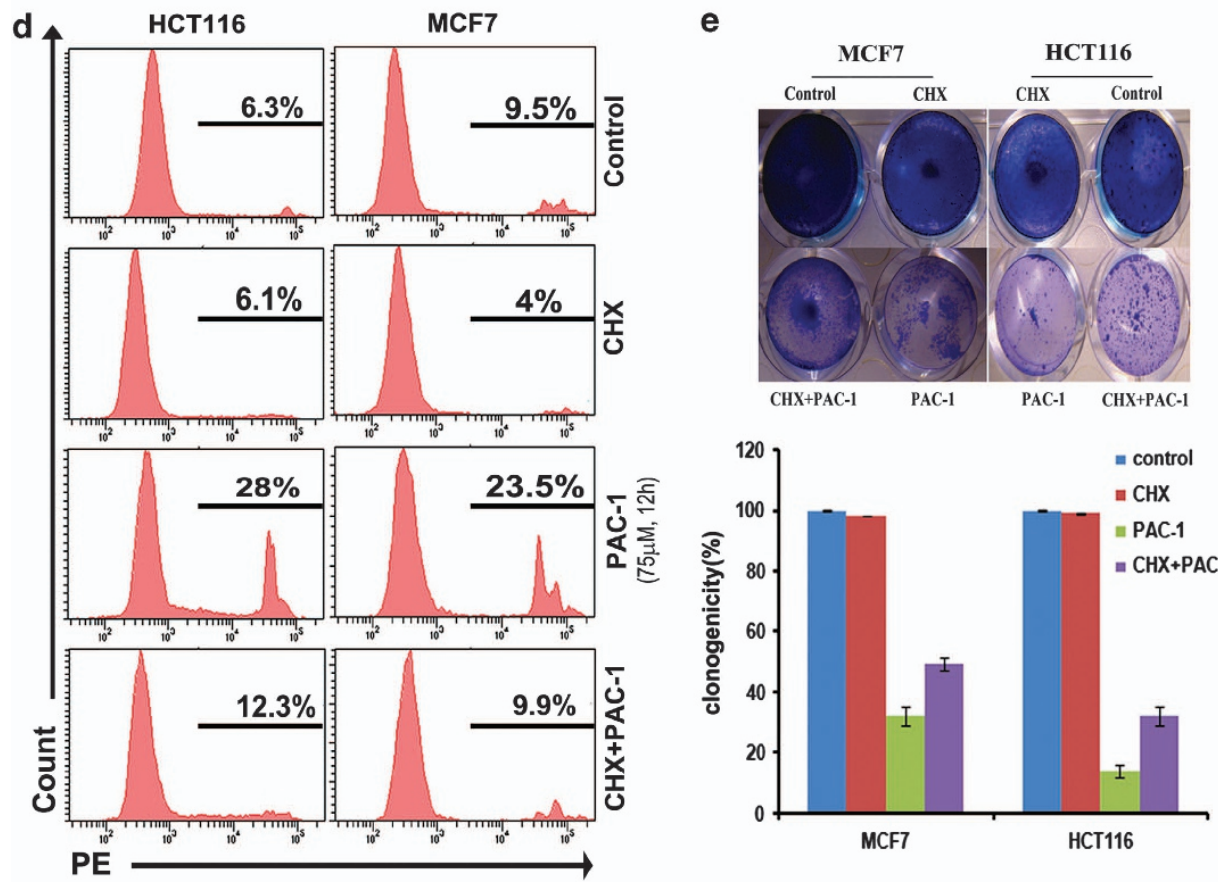
prolonged induction of Ero $1 \alpha$ by PAC-1 in multiple cell types including p53 $\mathrm{KO}$ cells. We also observed $\mathrm{CHOP}$ induction in treated cells; however, silencing of Ero1 $\alpha$ was more effective in reducing cell death and ER calcium release, pointing that induction of Ero1 $\alpha$ by PAC-1 contributes to ER stress. Prolonged induction of Ero1 $\alpha$ is able to oxidize ER lumen that may in turn enhance IP3-induced calcium release by disrupting the interaction between ERp44 and IP3R1. ${ }^{41}$ Consistent with this, a recent study revealed that overexpression of hyper-active, mutant Ero1 $\alpha$ leads to hyperoxidation of ER oxidoreductase ERp57, leading to UPR without the induction of antioxidant response. ${ }^{42}$ Our study also identified PUMA as a p53-independent regulator of mitochondrial permeabilization subsequent to Ero1 $\alpha$ mediated ER calcium release. BH3-only proteins such as Bim, Bik, Bid and PUMA have pivotal role in controlling apoptosis at the mitochondrial level by neutralizing antiapoptotic Bcl-2 family proteins and activating pro-apoptotic Bax/Bak proteins. ${ }^{43}$ Overexpression of transcriptional activator CHOP during ER stress leads to p53-independent upregulation of PUMA. ${ }^{44}$ Therefore, it is likely that upregulation of both Ero1 $\alpha$ at ER and PUMA at the mitochondria contributes to calcium leakage through MAM and both act as regulators of calcium leakage from ER to mitochondria. In accordance with this, we have noticed significant colocalization of Ero- $1 \alpha$ and PUMA in PAC-1-treated cells (data not shown). Supporting the essential role of PUMA, silencing of PUMA reduced cyt. $c$ release as well as apoptosis induced by PAC-1 in MCF7 cells. The ability of PAC-1 to induce MAMdependent ER calcium leakage into the mitochondria, generating a calcium and ROS overload at the mitochondria, could drive PUMA-dependant mitochondrial permeabilization independent of Bax/Bak. In agreement with this model, mitochondrial calcium uptake inhibitor significantly reduced cell death in Bax/Bak DKO cells.

Even though experiments described here identified Ero1 $\alpha$ as the key player of PAC-1-induced ER stress and subsequent cell death, further studies are required to understand how PAC-1 targets Ero1 $\alpha$. One of the potential functional targets identified for PAC-1 is its ability to chelate zinc, a metal necessary for the stability of diverse enzymes in ER lumen such as protein disulfide isomerase (PDI). ${ }^{45}$ Ero $1 \alpha$ oxidizes active-site cysteines of PDI, an enzyme critical for adding disulfide bonds into newly synthesized proteins. Because of its ability to maintain increased oxidizing equivalents, Ero1 $\alpha$ activity must be regulated to prevent excessive build up of ROS as well as hyper-oxidizing conditions in ER. Chelation of zinc by PAC- 1 appears to affect the activity of Ero $1 \alpha-P D I$ axis, leading to hyper-oxidation of ER, calcium release and build up of misfolded proteins at the ER. Ero1 $\alpha$, being an integral partner of MAM may further allow immediate calcium uptake by mitochondria leading to mitochondrial permeabilization. Overall, the results described revealed critical signaling involving Ero1 $\alpha$-dependent ER luminal hyper-oxidation and ER calcium leakage into the mitochondria through engagement of MAM, in PAC-1-induced cell death. Further studies may reveal whether Ero1 $\alpha$-dependent ER luminal hyperoxidation and mitochondrial calcium overload can be utilized as a possible target against cancer drug-resistance.

\section{Materials and Methods}

Cell lines and maintenance. MCF7, HeLa, SiHa, HCT116, SKOV3, U2OS, ADR-RES, T47D, MDAMB231, SW480 and HEK293 cells were maintained in DMEM containing $10 \%$ FBS in a humidified $\mathrm{CO}_{2}$ chamber at $37^{\circ} \mathrm{C}$. SV $40-$ immoratalized MEF WT and its variants were maintained in DMEM containing $10 \%$ FBS and antibiotics.

Chemicals and reagents. PAC- 1 was purchased from Calbiochem (EMD Millipore, Billerica, MA, USA). The other drugs such as MG132, thapsigargin, cisplatin, 3-methyladenine and cycloheximide were obtained from Sigma-Aldrich (St. Louis, MO, USA). The antibodies against GRP78 (E-4), GRP94 (H-212), Bak (G-23), Hsp90 (H-114), p53 (FL-393) were from Santa Cruz Biotechnology (Santa Cruz, CA, USA). Antibodies against caspase-3, cleaved caspase-3, caspase-7 caspase-2, CHOP (L63F7), LC3B, XIAP, GFP, Bcl-2, p-elF2 $\alpha$ (Ser51), elF2 $\alpha, \operatorname{IRE} 1 \alpha(14 \mathrm{C} 10)$, Ero1-L $\alpha$ and PUMA were obtained from Cell Signaling Technology (Beverly, MA, USA). Calnexin, Cyt. $c$ 6H2.B4 and H2AX antibodies were purchased from BD Pharmingen (San Diego, CA, USA).

Expression vectors and generation of stable cell lines. pcDNA3 caspase-3 was transfected in MCF7 using lipofectamine-LTX (Invitrogen, Carlsbad, CA, USA) and maintained in $800 \mu \mathrm{g} / \mathrm{ml}$ of G418 (Invitrogen). Single clones were selected for getting cell population with homogenous expression of caspase-3. Expression vectors for calcium sensor ratiometric FRET probe D1ER cameleon and Bcl-2 targeted at ER with cytochrome b5-targeting sequence ( $p c D N A 3$ Bcl-2Cb5-EGFP) were transfected into respective cells using lipofectamine. Cells expressing fluorophore were sorted out using FACS Aria II (BD Biosciences, Bedford, MA, USA). Cells expressing EGFP-LC3, cyt. c-EGFP and caspase-specific FRET probe CFP-DEVD-YFP were generated as reported previously. ${ }^{11,46}$ PUMA shRNA vector (NM_014417, Sigma-Aldrich) was transfected by means of lipofectamine-LTX and stable clones were selected by maintaining in $3 \mu \mathrm{g} / \mathrm{ml}$ of puromycin (Invitrogen). HeLa cells were stably transfected with expression vector for redox sensitive GFP targeted at mitochondria (roGFP-Mito) as described. ${ }^{36}$

Transfection of siRNA. HeLa and MCF7 cells were transfected with GRP78 siRNA, Ero1 $\alpha$ siRNA, CHOP siRNA or scrambled siRNA (Santa Cruz Biotechnology) according to manufacturer's protocol with recommended transfection reagent. The cells were replenished with fresh complete medium (DMEM containing $20 \%$ FBS) after $8 \mathrm{~h}$.

Cell death and functional assays. Western blot, clonogenicity assay and flow cytometry analyses for annexin binding, cell cycle status and caspase

\footnotetext{
Figure 3 GRP78-silencing enhances, whereas translational inhibitor cycloheximide reduces cell death mediated by PAC-1. (a and b) MCF7 and HeLa cells were transfected with siGRP78. Silencing of GRP78 was confirmed in blot. Cell death was analyzed by PI staining using FACS after silencing GRP78. The comparison and percentage of PI-positive cells are represented graphically $(n=3$, mean \pm S.D.). (c) ER stress was inhibited by using cycloheximide (CHX; $2 \mu \mathrm{g} / \mathrm{ml})$ along with PAC-1, and caspase activity was evaluated using FACS by employing caspase-3 and -7 specific FRET probe, CFP-DEVD-YFP, stably transfected in HeLa and MCF7 cells. Cleavage of DEVD sequence by activated caspases will cause FRET loss which is reflected as enhancement of CFP/YFP ratio. As shown in the gates (FRET lost-population), cycloheximide reduced percentage of cells with FRET loss, suggesting that ER stress inhibition reduces caspase activation induced by PAC-1. (d) Cell death was analyzed by PI staining using FACS in MCF7 and HCT116 after PAC-1 treatment in the presence and absence of cycloheximide. The percentage of PI-positive cells is indicated in respective histograms, suggesting that cycloheximide inhibited cell death induced by PAC-1. (e) Cell survival was analyzed by clonogenicity assay in PAC-1-treated MCF7 and HCT116 cells, with and without cycloheximide. As noted in images, translational inhibitor cycloheximide rescued cells from PAC-1-induced cell death when used along with PAC-1. Absorbance was measured for the solubilized stain and relative percentage of clonogenicity is represented in graph $(n=3$, mean \pm S.D. $)$
} 
a

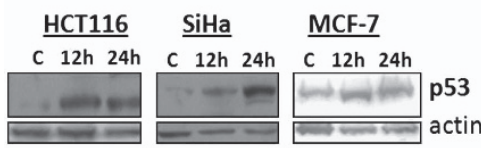

b

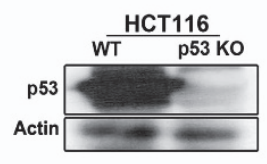

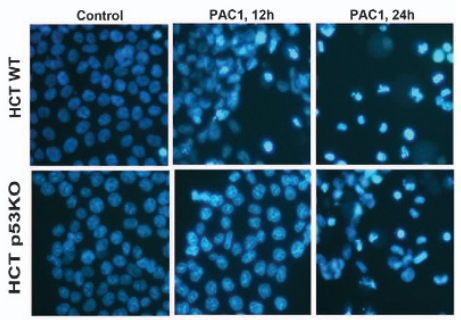

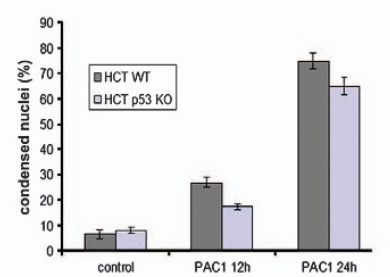

e
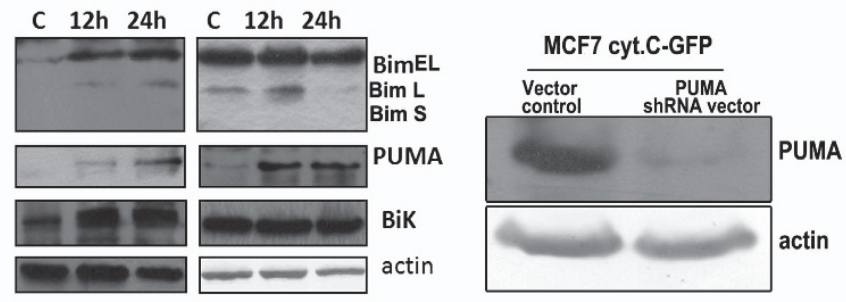

PUMA

f

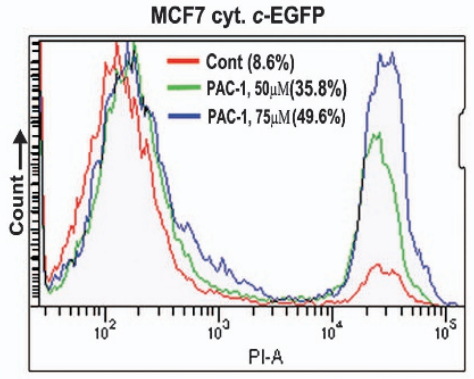

g

Merged

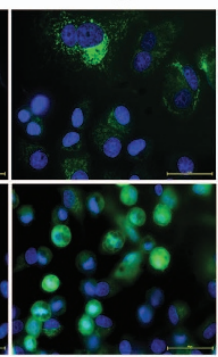

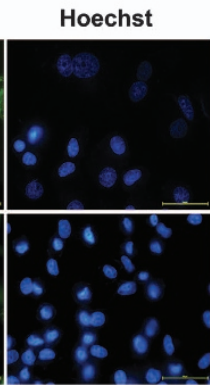

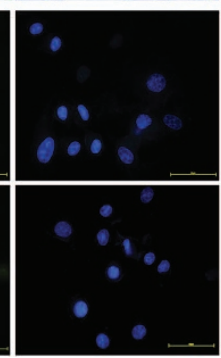

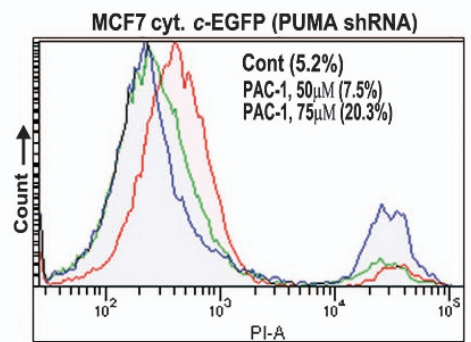
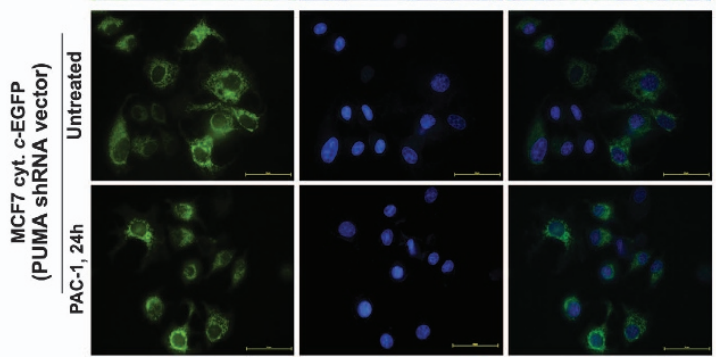

Figure 4 p53-independent PUMA upregulation by PAC-1 promotes mitochondria-mediated apoptosis. (a) Western blot analysis indicated that p53 was upregulated in multiple cancer cells upon PAC-1 treatment. (b) PAC-1 induced significant nuclear condensation in HCT116 and p53 KO HCT116 cells as seen in representative microscopic images and graph ( $n=3$, mean \pm S.D.). Western blot established the knockout status of p53 in HCT116. (c) Clonogenic cell survival assay revealed that PAC-1 effectively killed HCT116 p53 KO cells, although slightly lesser than its WT counterpart. Representative images and graph are given ( $n=3$, mean \pm S.D.). (d) PAC-1 induced upregulation of PUMA in multiple cancer cell lines. As seen in blot, PUMA is upregulated even in the absence of p53 (HCT116 p53 KO). Bim and Bik were also upregulated in SiHa cells but not in MCF7 and HCT116 cells, suggesting cell specificity of the response. (e) A representative western blot indicating silencing of PUMA in MCF7 cyt. $c$-EGFP cells. (f) Cell death was analyzed by PI staining using FACS in MCF7 cyt. $c$-EGFP cells as well as PUMA-silenced MCF7 cyt. $c$-EGFP cells. As noticed, silencing of PUMA inhibited PAC-1-induced cell death significantly. The percentages of PI-positive cells are mentioned. (g) Silencing of PUMA inhibited cyt. c release from the mitochondria and nuclear condensation in MCF7 cyt. c-EGFP cells upon PAC-1 treatment. The representative fluorescent microscopic images are shown (scale bar: $50 \mu \mathrm{m}$ ). The diffuse pattern of EGFP in cells represents release of cyt. $c$ from the mitochondria

activation in FRET probe-expressing cells were carried out as explained previously. ${ }^{11,47,48}$ For analysis of oxidation status of redox sensitive GFP, HeLa cells stably expressing the probe were treated with PAC-1 at $50 \mu \mathrm{M}$. After $12 \mathrm{~h}$, cells were trypsinized and analyzed by FACS at 405 and $488 \mathrm{~nm}$ laser lines, using $535 / 40$ emission filter in ratio mode. DTT was used as a reducing agent to validate the functionality of the probe as described. ${ }^{36}$ 
a

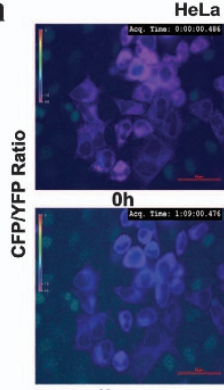

1h

b
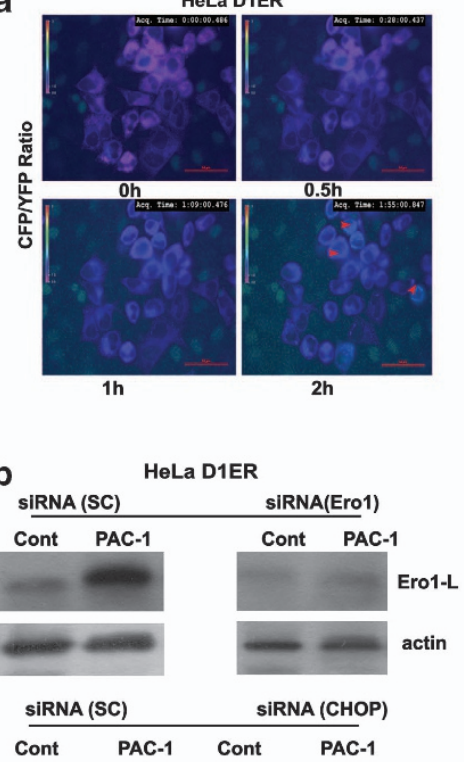

$2 \mathrm{~h}$

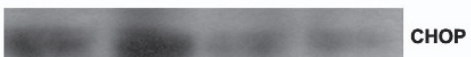

CHOP

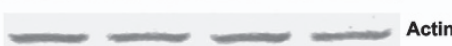

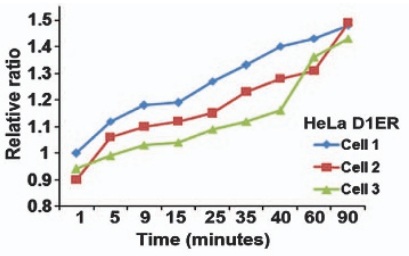

C 1.5

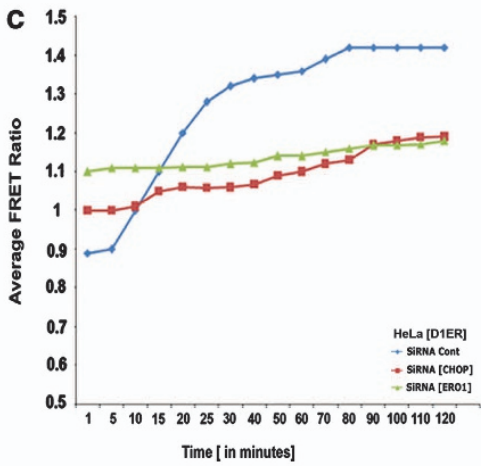

d 7

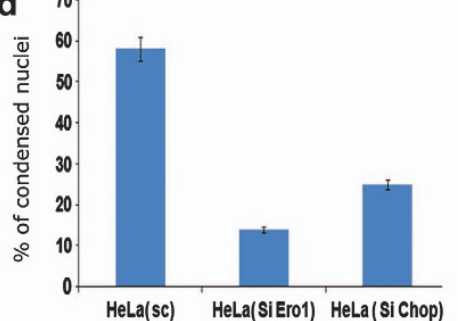

e
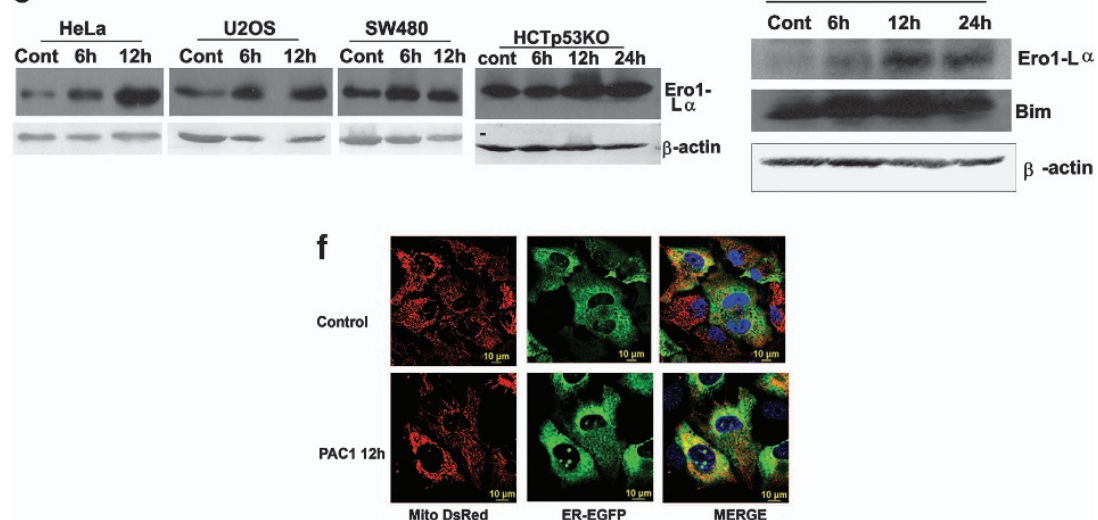

Figure 5 Ero $1 \alpha$ mediates calcium flux from ER to the mitochondria through engagement of MAM. (a) HeLa cells stably expressing ER-targeted ratiometric FRET probe (D1ER chameleon) was used to monitor release of ER calcium. CFP/YFP ratio was measured from images and represented graphically $(n=3)$. The ratio images of cells before PAC-1 treatment and $2 \mathrm{~h}$ after PAC-1 treatment are shown along with ratio scale. (b) HeLa D1ER cells were transfected with either scrambled siRNA or siRNA against human Ero $1 \alpha$ or CHOP as described. The cells were treated with PAC-1 for $24 \mathrm{~h}$ and whole-cell extract was used for western blot using Ero1 $\alpha$ or $\mathrm{CHOP}$ antibody. $\beta$-Actin served as loading control. (c) HeLa D1ER cells $24 \mathrm{~h}$ after transfections with siEro1 $\alpha$ or siCHOP were treated with PAC-1 and imaged for calcium release as described. The average CFP/YFP ratio was measured from images and represented graphically $(n=3)$. (d) Cells transfected with siSC (scrambled) or siEro1 $\alpha$ or siCHOP were treated with PAC-1 for $24 \mathrm{~h}$. Cell death was quantified after staining the cells using Hoechst to visualize chromatin condensation. (e) HeLa, U2OS, SW480, p53 knockout HCT116 cells and MEF Bax/Bak DKO cells were treated with PAC-1 for indicated time periods. The whole-cell extract was prepared and used for western blotting using antibody against Ero1 $\alpha$. Induction of Bim exhibited by MEF Bax/Bak DKO cells is also shown. $\beta$-Actin served as loading control. (f) HeLa cells expressing EGFP targeted at ER and DsRed targeted at mitochondria were treated with PAC-1 for $12 \mathrm{~h}$. Representative confocal images of mitochondria and ER of treated and untreated cells are shown

Analysis of XBP1 mRNA splicing by RT-PCR. RNA was isolated from cells using Trizol (Invitrogen) and $2 \mu \mathrm{g}$ each of RNA was used for cDNA synthesis using MMLV-reverse transcriptase (Promega, Madison, WI, USA). Expression analysis of XBP1 mRNA splicing was performed after normalizing with endogenous control, $\beta$-actin. The amplicons were resolved using $3 \%$ agarose gel. Primer sequences are mentioned in Supplementary Information.
Analysis of ER $\mathrm{Ca}^{2+}$ release by fluorescent microscopic timelapse imaging. Time-lapse imaging was carried out in a live-cell incubation chamber (Tokai Hit, Fujinomiya-shi Shizuoka-ken, Japan) that maintains optimum $\mathrm{CO}_{2}$, temperature and humidity, using motorized inverted fluorescence microscope Nikon Eclipse TE2000-E (Nikon Instruments Inc., Melville, NY, USA) equipped with a CARV II Confocal-imager (BD Biosciences). Images were captured using 
a

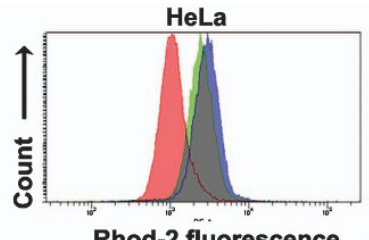

Rhod-2 fluorescence

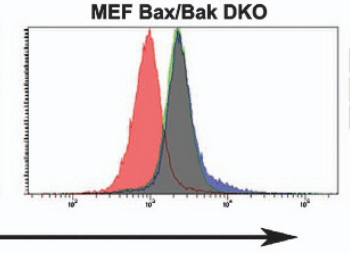

MEF Bax/Bak DKO

b

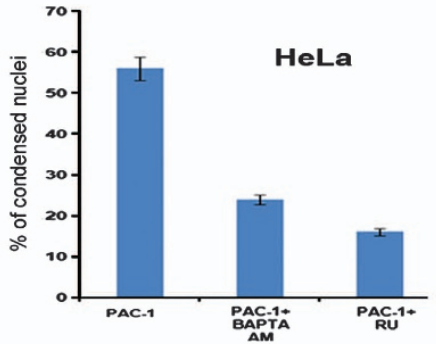

Control

PAC $-1[50 \mu \mathrm{M}]$

PAC-1 $[100 \mu \mathrm{M}]$

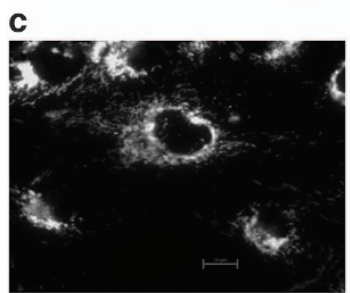

HeLa roGFP-Mito

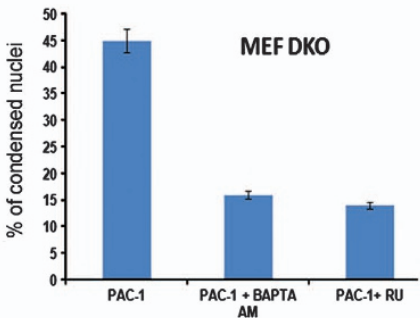

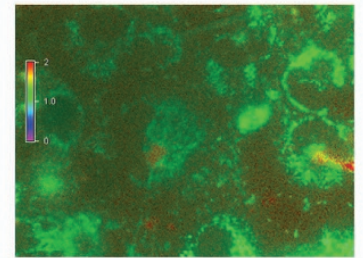

Control

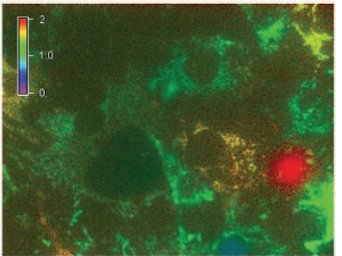

PAC-1

Ratio image of $405 / 490 \mathrm{~nm}$ channel

d

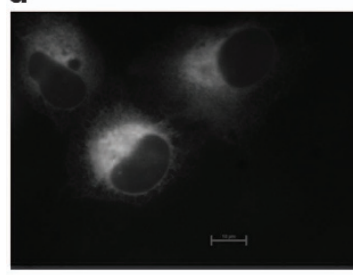

HeLa roGFP2-iL-KDEL

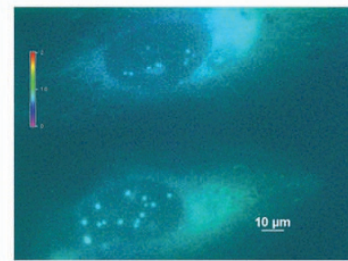

Control

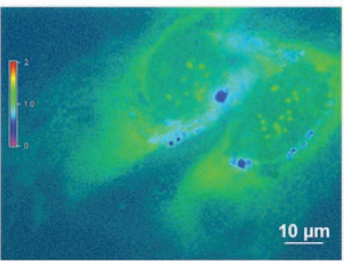

PAC-1

Ratio image of $405 / 490 \mathrm{~nm}$ channel

Figure 6 ER hyper-oxidation, mitochondrial calcium uptake and mitochondrial ROS generation contributes to cell death by PAC-1. (a) HeLa and MEF DKO cells were treated with 50 or $100 \mu \mathrm{M}$ of PAC-1 for $24 \mathrm{~h}$. The cells were stained with mitochondrial calcium indicator Rhod-2 as described. Rhod-2 fluorescence analyzed by FACS is shown as histograms. (b) HeLa and MEF DKO cells were either pretreated with $0.05 \%$ DMSO or mitochondrial calcium uptake inhibitor, Ru360 ( $25 \mu \mathrm{M})$ or cell-permeant calcium chelator, BAPTA-AM $(50 \mu \mathrm{M})$ followed by PAC-1 treatment for $24 \mathrm{~h}$. Cell death was quantified by chromatin condensation assay as described $(n=3$, mean \pm S.D.). (c) HeLa cells stably expressing mitochondria-targeted roGFP were developed as described. Microscopic image of mitochondrial localization of the probe is shown at $\times 100$ magnification. The emission at $535 / 30 \mathrm{~nm}$ was collected at dual excitation $405 / 20 x$ and $490 / 20 x$ in sequential mode using $\times 60$ objective for both untreated and treated (PAC1 for $12 \mathrm{~h}$ ) stable cells. The ratio images were generated by dividing $405 \mathrm{~nm}$ channel by $490 \mathrm{~nm}$ channel on a pixel by pixel basis. The ratio scale is also shown. (d) HeLa cells stably expressing ER-targeted roGFP, roGFP2-iL-KDEL was developed as described. Microscopic image of ER-targeted probe is shown at $\times 100$ magnification. Ratio imaging was carried out as earlier using $\times 100$ objectives. The ratio images were generated by dividing $405 \mathrm{~nm}$ channel by $490 \mathrm{~nm}$ channel on a pixel by pixel basis. The ratio scale is also shown

EMCCD camera Andor iXON 885 (Andor Technology PIc., Belfast, BT12 7AL, UK) and IPLab software (BD Biosciences) at regular intervals. To minimize photobleaching, the intensity of light was reduced to $1-4 \%$ by intensity control. Mean intensity of fluorescent cell was measured after ROI selection and background subtraction using IPLab software. For cameleon ratio-imaging, cells expressing the probes were maintained in chambered cover glass and imaged for $30 \mathrm{~min}$ after addition of drugs at an interval of $200 \mathrm{~ms}$. CFP was excited with $438 \pm 12 \mathrm{~nm}$ band-pass filter and dual emission was collected at $480 \pm 15 \mathrm{~nm}$ and $542 \pm 27 \mathrm{~nm}$ band-pass filter in ratio mode. Analysis of FRET-ratio was done by NIS-Elements software (Nikon Instruments Inc.).

Analysis of MAM by fluorescent microscopy and confocal imaging. Cells stably expressing DsRed targeted at the mitochondria (Mito DsRed) and EGFP targeted at ER (ER-EGFP) (Clontech Laboratories Inc.,
Mountain View, CA, USA) were used for visualizing MAM by high magnification imaging. For imaging by fluorescent microcopy, cells were grown on glass bottom chambered cover glass and imaging was carried out with Nikon Eclipse TiE microscope (Nikon Instruments Inc.) using $\times 601.4$ NA objective. For confocal imaging, cells were seeded on glass bottom plates and imaged using $\times 631.4 \mathrm{NA}$ objective. Confocal imaging was carried out using A1R Laser Scanning microscope (Nikon Instruments Inc.).

Redox sensitive GFP imaging. For imaging of redox sensitive GFP, cells were maintained in chambered cover glass and imaged under epifluorescent microscope using Xenon as the exciation light source (Lamda XL; Sutter Instrument Company, Novato, CA, USA). The emission wavelenght $535 / 30 \mathrm{~nm}$ was collected at dual excitation using filter set 405/20x and 490/20x in sequential mode. Images were captured using CCD camera (Cool Snap HQ; Photometrics, 
Tucson, AZ, USA). The ratio images were generated by dividing $405 \mathrm{~nm}$ channel by $490 \mathrm{~nm}$ channel on a pixel by pixel basis using NIS element software.

\section{Conflict of Interest}

The authors declare no conflict of interest.

Acknowledgements. Immoratalized MEF wild-type and Bax/Bak DKO cells were kindly provided by Dr. Stanely J Korsemeyer (Harvard Medical School, Boston, MA). MEF WT and its Apaf- $1 \mathrm{KO}$ counterpart cells were provided by Dr. Tak W. Mak (Ontario Cancer Institute, Canada). HCT116 WT and its p53 KO cells were provided by Dr. Bert Vogelstein (John Hopkins School of Medicine, Baltimore, USA). The expression vector, $\mathrm{Bcl}-2$ targeted at $\mathrm{ER}$ with cytochrome b5-targeting sequence (pcDNA3 Bcl-2Cb5-EGFP) was a kind gift by Dr. Clark Distelhorst. Plasmids containing calcium sensor ratiometric FRET probe D1ER cameleon and FRET probe pcDNA3 DEVD (ECFP-DEVD-YFP) were kindly provided by Dr. Roger $Y$ Tsien (HHMIL, University of California, San Diego) and Dr. Gavin Welsh (University of Bristol, UK) respectively. pEGFP-LC3 was a kind gift by Dr. Tamotsu Yoshimori and Dr. Noboru Mizushima, Tokyo Medical and Dental University, Tokyo. RoGFPMito was kindly supplied by Dr. S James Remington (University of California San Diego, La Jolla, CA, USA). ER-targeted redox sensitive GFP (pcDNA3.1 roGFP2-iLKDEL) was provided Dr. Neil J Bulleid (University of Glasgow, Glasgow, UK). This study was supported by grants from the Innovative Young Biotechnologist Award, Department of Biotechnology (DBT), Government of India and Research fellowship from University Grants Commission (UGC) to MS, Indian Council of Medical Research (ICMR) to PKS and KAM and Council of Scientific and Industrial Research (CSIR) to JJ. We thank Mrs Indu Ramachandran and Mr Anurup KG for support in FACS and confocal imaging.

1. Earnshaw WC, Martins LM, Kaufmann SH. Mammalian caspases: structure, activation, substrates, and functions during apoptosis. Ann Rev Biochem 1999; 68 383-424.

2. Cohen GM. Caspases: the executioners of apoptosis. Biochem J 1997; 326(Pt 1): 1.

3. Yip KW, Reed JC. Bcl-2 family proteins and cancer. Oncogene 2008; 27 6398-6406.

4. Thompson CB. Apoptosis in the pathogenesis and treatment of disease. Science 1995 267: $1456-1462$.

5. Kuwana T, Newmeyer DD. Bcl-2-family proteins and the role of mitochondria in apoptosis. Curr Opin Cell Biol 2003; 15: 691-699.

6. Chelur DS, Chalfie M. Targeted cell killing by reconstituted caspases. Proc Natl Acad Sci USA 2007; 104: 2283.

7. Zorn JA, Wells JA. Turning enzymes ON with small molecules. Nat Chem Biol 2010; 6 179-188.

8. Putt KS, Chen GW, Pearson JM, Sandhorst JS, Hoagland MS, Kwon JT et al. Smallmolecule activation of procaspase-3 to caspase-3 as a personalized anticancer strategy. Nat Chem Biol 2006; 2: 543-550.

9. Peterson QP, Goode DR, West DC, Ramsey KN, Lee JJY, Hergenrother PJ. PAC-1 activates procaspase-3 in vitro through relief of zinc-mediated inhibition. J Mol Biol 2009; 388: $144-158$.

10. Peterson QP, Hsu DC, Novotny CJ, West DC, Kim D, Schmit JM et al. Discovery and canine preclinical assessment of a nontoxic procaspase-3-activating compound. Cancer Res 2010; 70: 7232-7241.

11. Seervi M, Joseph J, Sobhan PK, Bhavya BC, Santhoshkumar TR. Essential requirement of cytochrome $\mathrm{c}$ release for caspase activation by procaspase-activating compound defined by cellular models. Cell Death Dis 2011; 2: e207.

12. Peterson QP, Hsu DC, Goode DR, Novotny CJ, Totten RK, Hergenrother PJ. Procaspase-3 activation as an anti-cancer strategy: structure-activity relationship of procaspase-activating compound 1 (PAC-1) and its cellular co-localization with caspase-3. J Med Chem 2009; 52: 5721-5731.

13. Dasika GK, Lin SC, Zhao S, Sung P, Tomkinson A, Lee EY. DNA damage-induced cell cycle checkpoints and DNA strand break repair in development and tumorigenesis. Oncogene 1999; 18: 7883-7899.

14. Klionsky DJ, Cuervo AM, Seglen PO. Methods for monitoring autophagy from yeast to human. Autophagy 2007; 3: 181-206.

15. Szegezdi E, Logue SE, Gorman AM, Samali A. Mediators of endoplasmic reticulum stress-induced apoptosis. EMBO Rep 2006; 7: 880-885.

16. Li J, Lee AS. Stress induction of GRP78/BiP and its role in cancer. Curr Mol Med 2006; 6 : $45-54$.

17. Xing X, Li Y, Liu H, Wang L, Sun L. Glucose regulated protein 78 (GRP78) is overexpressed in colorectal carcinoma and regulates colorectal carcinoma cell growth and apoptosis. Acta Histochem 2011; 113: 777-782.
18. Ferri KF, Kroemer G. Organelle-specific initiation of cell death pathways. Nat Cell Biol 2001; 3: E255-E263.

19. Wang NS, Unkila MT, Reineks EZ, Distelhorst CW. Transient expression of wild-type or mitochondrially targeted $\mathrm{Bcl}-2$ induces apoptosis, whereas transient expression of endoplasmic reticulum-targeted $\mathrm{Bcl}-2$ is protective against Bax-induced cell death. J Biol Chem 2001; 276: 44117-44128.

20. Rudner J, Lepple-Wienhues A, Budach W, Berschauer J, Friedrich B, Wesselborg S et al. Wild-type, mitochondrial and ER-restricted Bcl-2 inhibit DNA damage-induced apoptosis but do not affect death receptor-induced apoptosis. J Cell Sci 2001; 114: $4161-4172$

21. Chandrika BB, Maney SK, Lekshmi SU, Retnabhai ST. Endoplasmic reticulum targeted Bcl2 confers long term cell survival through phosphorylation of heat shock protein 27. Int J Biochem Cell Biol 2010; 42: 1984-1992.

22. Li J, Lee B, Lee AS. Endoplasmic reticulum stress-induced apoptosis. J Biol Chem 2006; 281: $7260-7270$.

23. Rao RV, Ellerby HM, Bredesen DE. Coupling endoplasmic reticulum stress to the cell death program. Cell Death Diff 2004; 11: 372-380.

24. Puthalakath $H$, O'Reilly LA, Gunn P, Lee L, Kelly PN, Huntington ND et al. ER stress triggers apoptosis by activating BH3-only protein Bim. Cell 2007; 129: 1337-1349.

25. Jeffers JR, Parganas E, Lee $\mathrm{Y}$, Yang $\mathrm{C}$, Wang JL, Brennan J et al. Puma is an essential mediator of p53-dependent and-independent apoptotic pathways. Cancer Cell 2003; 4: 321-328

26. Orrenius S, Zhivotovsky B, Nicotera P. Regulation of cell death: the calcium-apoptosis link. Nat Rev Mol Cell Biol 2003; 4: 552-565.

27. Rowland AA, Voeltz GK. Endoplasmic reticulum-mitochondria contacts: function of the junction. Nat Rev Mol Cell Biol 2012; 13: 607-625.

28. Palmer AE, Jin C, Reed JC, Tsien RY. Bcl-2-mediated alterations in endoplasmic reticulum $\mathrm{Ca} 2+$ analyzed with an improved genetically encoded fluorescent sensor. Proc Natl Acad Sci USA 2004; 101: 17404-17409.

29. Mathai JP, Germain M, Shore GC. BH3-only BIK regulates BAX,BAK-dependent release of $\mathrm{Ca} 2+$ from endoplasmic reticulum stores and mitochondrial apoptosis during stress-induced cell death. J Biol Chem 2005; 280: 23829-23836.

30. Rodriguez DA, Zamorano S, Lisbona F, Rojas-Rivera D, Urra H, Cubillos-Ruiz JR et al. $\mathrm{BH} 3-$ only proteins are part of a regulatory network that control the sustained signaling of the unfolded protein response sensor IRE1 $\alpha$. EMBO J 2012; 31: 2322-2335.

31. Li G, Mongillo M, Chin KT, Harding H, Ron D, Marks AR et al. Role of ERO1-alphamediated stimulation of inositol 1,4,5-triphosphate receptor activity in endoplasmic reticulum stress-induced apoptosis. J Cell Biol 2009; 186: 783-792.

32. Chin KT, Kang G, Qu J, Gardner LB, Coetzee WA, Zito E et al. The sarcoplasmic reticulum luminal thiol oxidase ERO1 regulates cardiomyocyte excitation-coupled calcium release and response to hemodynamic load. FASEB J 2011; 25: 2583-2591.

33. Gilady SY, Bui M, Lynes EM, Benson MD, Watts R, Vance JE et al. Ero1alpha requires oxidizing and normoxic conditions to localize to the mitochondria-associated membrane (MAM). Cell Stress Chaperones 2010; 15: 619-629.

34. Anelli T, Bergamelli L, Margittai E, Rimessi A, Fagioli C, Malgaroli A et al. Ero1alpha regulates $\mathrm{Ca}(2+)$ fluxes at the endoplasmic reticulum-mitochondria interface (MAM). Antioxid Redox Signal 2012; 16: 1077-1087.

35. Hayashi T, Rizzuto R, Hajnoczky G, Su TP. MAM: more than just a housekeeper. Trends Cell Biol 2009; 19: 81-88.

36. Hanson GT, Aggeler R, Oglesbee D, Cannon M, Capaldi RA, Tsien RY et al. Investigating mitochondrial redox potential with redox-sensitive green fluorescent protein indicators. J Biol Chem 2004; 279: 13044-13053.

37. van Lith M, Tiwari S, Pediani J, Milligan G, Bulleid NJ. Real-time monitoring of redox changes in the mammalian endoplasmic reticulum. J Cell Sci 2011; 124(Pt 14): 2349-2356.

38. West DC, Qin Y, Peterson QP, Thomas DL, Palchaudhuri R, Morrison KC et al. Differential effects of procaspase- 3 activating compounds in the induction of cancer cell death. $\mathrm{Mol}$ Pharm 2012; 9: 1425-1434.

39. Fribley A, Wang CY. Proteasome inhibitor induces apoptosis through induction of endoplasmic reticulum stress. Cancer Biol Ther 2006; 5: 745-748.

40. Tsai YC, Weissman AM. Ubiquitylation in ERAD: Reversing to Go Forward? PLoS Biol 2011; 9: e1001038

41. Tavender TJ, Bulleid NJ. Molecular mechanisms regulating oxidative activity of the Ero1 family in the endoplasmic reticulum. Antioxid Redox Signal 2010; 13: 1177-1187.

42. Hansen HG, Schmidt JD, Soltoft CL, Ramming T, Geertz-Hansen HM, Christensen B et al. Hyperactivity of the Ero1alpha oxidase elicits endoplasmic reticulum stress but no broad antioxidant response. J Biol Chem 2012; 287: 39513-39523.

43. Willis SN, Adams JM. Life in the balance: how BH3-only proteins induce apoptosis. Curr Opi Cell Biol 2005; 17: 617-625.

44. Cazanave SC, Elmi NA, Akazawa Y, Bronk SF, Mott JL, Gores GJ. CHOP and AP-1 cooperatively mediate PUMA expression during lipoapoptosis. Am J Physiol Gastrointest Liver Physiol 2010; 299: G236-G243.

45. Solovyov A, Gilbert HF. Zinc-dependent dimerization of the folding catalyst, protein disulfide isomerase. Protein Sci 2004; 13: 1902-1907.

46. Chandrika BB, Maney SK, Lekshmi SU, Joseph J, Seervi M, SP K et al. Bax deficiency mediated drug resistance can be reversed by endoplasmic reticulum stress induced death signaling. Biochem Pharmacol 2010; 79: 1589-1599. 
47. Joseph J, Seervi M, Sobhan PK, Retnabai ST. High throughput ratio imaging to profile caspase activity: potential application in multiparameter high content apoptosis analysis and drug screening. PLoS One 2011; 6: e20114.

48. Achuthan S, Santhoshkumar TR, Prabhakar J, Nair SA, Pillai MR. Drug-induced senescence generates chemoresistant stemlike cells with low reactive oxygen species. J Biol Chem 2011; 286: 37813-37829.
Cell Death and Disease is an open-access journal published by Nature Publishing Group. This work is licensed under a Creative Commons Attribution-NonCommercialNoDerivs 3.0 Unported License. To view a copy of this license, visit http://creativecommons.org/licenses/by-nc-nd/3.0/

Supplementary Information accompanies this paper on Cell Death and Disease website (http://www.nature.com/cddis) 